\title{
Grado de satisfacción y preferencias técnico-tácticas de jugadores de balonmano en etapas de formación según el sexo de los jugadores
}

\author{
Level of satisfaction and technical-tactical preferences of \\ handball players in formative stages by gender of players
}

\section{Grau de satisfação e preferências técnico-táticas dos jogadores de andebol em etapas de formaçáo consoante o sexo dos jogadores}

\author{
Antonio García-Angulo ${ }^{1,2}$, Francisco García-Ángulo y Enrique Ortega Toro ${ }^{1 *}$ \\ 1 Facultad de Ciencias del Deporte. Universidad de Murcia. Campus de Excelencia Internacional Regional "Campus Mare Nostrum", España. \\ 2 Federación de Fútbol de la Región de Murcia (FFRM), España.
}

Resumen: Los objetivos del presente estudio son:a) analizar las preferencias técnico-tácticas de jugadores de balonmano, tanto en entrenamiento como en competición, diferenciando entre sexos; b) Saber cuáles son las acciones preferidas de los jugadores de balonmano diferenciando entre sexos; y c) diferenciar las situaciones de igualdad o desigualdad numérica de juego preferidas por los jugadores según el sexo del jugador. Se desarrolló un estudio de carácter selectivo sobre los jugadores de categoría infantil y cadete que participaron en el Campeonato de Espańa de Balonmano por selecciones territoriales de 2012 ( $n=1015)$, diferenciando dos grupos: a) masculino y b) femenino. Se utilizó como instrumento el "Cuestionario de Satisfacción y Preferencias en Jugadores de Balonmano". Los resultados más importantes mostraron que: a) La acción técnico-táctica favorita en ambos grupos es la de lanzar; b) La fase del juego favorita en ambos grupos es la de atacar; c) las chicas muestran una mayor preferencia por la defensa que los chicos; $y$ d) Ciertas acciones defensivas ocasionan una gran satisfacción en ambos grupos, algo que se cree condicionado por lo que creen que les gusta y que piden sus entrenadores. Estos datos pueden servir para optimizar el proceso de enseñanza-aprendizaje del balonmano en chicos y chicas, entendiendo que sus preferencias no son las mismas.

Palabrasclave:enseñanzadeportiva; orientación demeta;autodeterminación; balonmano.

Abstract: The aims of this study are:a) analyse the technical and tactical preferences of handball players in both training and competition, differentiating between genders; b) learn which are the preferred actions of handball players, differentiating between genders; c) differentiate the numerical situations of play preferred by the players according to gender of the player. A selective study was carried out on players in the U-14 and U-16 categories who participated in Spain's 2012 Handball Championship for territorial teams ( $\mathrm{n}=1015)$, dividing them into two groups: $\mathrm{a})$ male and $\mathrm{b}$ ) female. The methodology used was the "Handball Player Satisfaction and Preferences
Questionnaire". The most important results showed that: a) the technicaltactical action preferred for both groups is to launch the ball; b) the game phase preferred for both groups is attack; c) girls show a greater preference for defence than boys; d) some defensive actions cause great satisfaction in both groups, while some feel themselves conditioned by what they believe their coaches like and ask for. These data can be used to optimise the process of teaching and learning in boys' and girls' handball, understanding that their preferences are not the same.

Key Words: sport education;achievement goals; self-determination; handball. Resumo: Os objetivos do presente estudo são: a) Analisar as preferências técnico-táticas dos jogadores de andebol, tanto a nível de treino como de competiçáo, com diferenciaçáo entre sexos; b) Saber quais são as açóes preferidas dos jogadores de andebol com diferenciação entre sexos; e c) diferenciar as situaçôes numéricas de jogo preferidas pelos jogadores consoante o sexo do jogador. Foi desenvolvido um estudo de carácter seletivo sobre os jogadores de categoria sub-14 e sub-16 que participaram no Campeonato de Espanha de Andebol por seleçōes territoriais de 2012 $(\mathrm{n}=1015)$, diferenciando dois grupos: a) masculino e b) feminino. O instrumento utilizado foi o "Questionário de Satisfação e Preferências de Jogadores de Andebol". Os resultados mais importantes mostraram que: a) A ação técnico-tática favorita em ambos os grupos é a de lançar; b) A fase do jogo favorita em ambos os grupos é a de atacar; c) As raparigas mostram maior preferência pela defesa que os rapazes; e d) Determinadas açôes defensivas proporcionam grande satisfação em ambos os grupos, algo que se julga condicionado pelo que acreditam agradar aos seus treinadores ou o que lhes é pedido por estes. Estes dados poderão servir para otimizar o processo de ensino-aprendizagem do andebol em rapazes e raparigas, e para compreender que as suas preferências não são as mesmas.

Palavras-chave:ensino desportivo; orientaçáo para uma meta; autodeterminação; andebol.

\section{Introducción}

Uno de los aspectos fundamentales que garantizan una actitud positiva y una mayor adherencia a la práctica deportiva

Dirección para correspondencia [Correspodence address]: Enrique Ortega Toro. E-mail: eortega@um.es es que el joven deportista se encuentre motivado y satisfecho, siendo el elemento fundamental con el que cuentan los formadores deportivos el adaptar sus sesiones de trabajo a las preferencias técnico-tácticas de sus jugadores (Borrego, Ca- 
rrillo, y Díaz, 2014; Chen, 2001; González, Donolo, Rinaudo, y Paoloni, 2011; Treasure y Roberts, 2001).

En este sentido, la motivación es un elemento esencial en el desarrollo deportivo que se verá afectada por una serie de variables personales, ambientales y sociales. Y que determinará el tipo de práctica deportiva, la adherencia a esa práctica, la intensidad con la que se realiza la sesión y finalmente, el rendimiento deportivo del deportista (Balaguer, Castillo, y Duda, 2007).

El uso de la motivación como un elemento esencial para la adherencia a la práctica deportiva se justifica mediante la Teoría de las Metas de logro (Nicholls, 1989), que intenta analizar cómo inciden los diferentes procesos psicológicos individuales y colectivos en el desarrollo de la iniciación deportiva (Cervelló, Escartí, y Guzmán, 2007; Santos-Rosa, García, Jiménez, Moya, y Cervelló, 2007) y la Teoría de la autodeterminación (Deci y Ryan, 1985; 2000)que considera que al cubrir con las necesidades psicológicas básicas de autonomía, competencia y relación con el resto de jugadores del equipo se va a repercutir positivamente en el grado de motivación del joven deportista, suministrando además una sensación de competencia que repercutirá favorablemente en la motivación intrínseca (Balaguer, Castillo, y Duda, 2007; 2008).

En base a estas teorías, no todos los jugadores se desarrollan de una misma forma, siendo el grado de evolución y el sexo uno de los aspectos que diferenciará los intereses que muestran los jóvenes jugadores en cada etapa. Esto es debido a que estos intereses evolucionan a situaciones más complejas conforme el grado madurativo y la experiencia de los jugadores de balonmano es mayor (García-Angulo, Ortega, y Mendoza, 2014; Ortega, García-Angulo, Mendoza, y López, 2015; Ortega, Palao, Sainz de Baranda, y García, 2009).

Estas diferencias se acentúan entre sexos, ya que el grado evolutivo, intereses y motivaciones no son los mismos en niños y niñas. Será labor de los entrenadores el desarrollar los principios de adaptación y progresión, con el fin de elaborar unas sesiones que logren el desarrollo de la motivación en la práctica (Cárdenas, 2003; Giménez y Sáenz-López, 2006; 2007; Ortega, Piñar, y Cárdenas, 1999).

Realizando una comparativa entre sexos se encuentran diferencias en los aspectos motivacionales, siendo la tendencia general que las mujeres tienen una mayor orientación hacia la tarea, mientras que los hombres hacia el ego (Castillo, Balaguer, y Duda, 2000; Cecchini, González, y Montero, 2008; Gano-Overway y Duda, 2001; Hanrahan yBiddle, 2002; Hanrahan yCerin, 2009; Kavussanu y Roberts, 2001).

En esta línea, una serie de trabajos muestran una mayor predisposición a la motivación intrínseca por parte de las mujeres frente a una mayor motivación extrínseca y orientación al ego por parte de los hombres (Alonso, Martínez-Galindo,
Moreno, y Cervelló, 2005; Chantal, Guay, Dobreva-Martinova, y Vallerand, 1996; González y Martínez, 2015; MartínezGalindo, Alonso, Cervelló, y Moreno 2009; Moreno,Hellín, Hellín, y Cervelló 2006; Petherick y Weigand, 2002).

Por todo ello, y debido a las diferencias existentes en los factores que ocasionan motivación y satisfacción entre sexos, y a la importancia de cumplir con las preferencias de los jóvenes deportistas, los objetivos del presente trabajo son: a) analizar las preferencias técnico-tácticas de jugadores de balonmano, tanto en entrenamiento como en competición, diferenciando entre sexos; b) Saber cuáles son los ejercicios preferidos de los jugadores de balonmano diferenciando entre sexos; y c) diferenciar las situaciones de igualdad o desigualdad numérica de juego preferidas por los jugadores según el sexo del jugador.

\section{Método}

\section{Participantes}

La muestra objeto de estudio fueron 1015 jugadores de balonmano en etapas de formación: hombres $(\mathrm{n}=505)$ y mujeres $(n=510)$, que participaban en el Campeonato de España de Balonmano por Comunidades Autónomas en categoría infantil y cadete (13-16 años).

\section{Instrumento}

Se empleó como instrumento el "Cuestionario de Satisfacción y Preferencias en Jugadores de Balonmano"(incluido en anexo 1). Tal instrumento es una adaptación del "Cuestionario de Satisfacción y Preferencias en Jugadores de Baloncesto" (CSPJB) diseñado y validado por Ortega, Giménez, Palao, y Sainz de Baranda (2008).

El instrumento CSPJB tuvo unos valores de fiabilidad aceptables, en concreto ocho ítems tuvieron valores entre 0.41-0.61, treinta y tres entre $0.61-0.8$ y quince con valores superiores a 0.8 . En el presente estudio, el ítem que menor fiabilidad obtuvo fue el de "Acción técnico-tácticaindividual en partido", con una fiabilidad de 0.82 .

\section{Procedimiento}

Para el registro de los datos, una vez la aprobación de la comisión ética de la Universidad de Murcia, se contactó con la Federación Española de Balonmano. Se utilizó el campeonato de España de Selecciones autonómicas Infantiles y Cadetes para el registro de los datos. Los datos fueron registrados en una sala, en la que los jugadores sin los entrenadores, rellenaban el cuestionario. 


\section{Análisis estadístico}

Se desarrolló un estudio de carácter selectivo. Para el análisis estadístico se utilizó el software SPSS (Statistical Pack agefor the Social Sciences) de IBMC en su versión 21.0. Se desarrolló un análisis descriptivo usando recuento de frecuencias y porcentaje para las variables categóricas y los descriptivos media y desviación típica para las variables continuas. Para el análisis inferencial se utilizó la prueba de Chi-cuadrado( $\chi 2)$, utilizando la $\mathrm{V}$ de Cramer para valorar la fuerza en la relación
$(\Phi)$, y la prueba estadística U de Mann-Whitney. Todos los datos se trataron con un nivel de significación $\rho<.05$.

\section{Resultados}

\section{Preferencias en competición y entrenamiento}

Los resultados hallados en la tabla 1 muestran las preferencias técnico-tácticas que tienen los jugadores en competición y entrenamiento, y su preferencia por atacar o defender.

Tabla 1. Preferencias sobre ataque y defensa, y en acciones técnico-tácticas individuales en partido y en entrenamiento.

\begin{tabular}{|c|c|c|c|c|c|}
\hline Variable & Categoría & Masculino & Femenino & Total & $\begin{array}{c}\rho \text { valor } \\
\left(\chi^{2}\right)\end{array}$ \\
\hline \multirow{5}{*}{ Acción técnico-táctica individual en partido } & Lanzar & $50.5 \%$ & $38.5 \%$ & $44.4 \%$ & \multirow{5}{*}{$\begin{array}{c}.010^{* *} \\
\Phi=.061\end{array}$} \\
\hline & Fintar & $16.4 \%$ & $17.5 \%$ & $17 \%$ & \\
\hline & Pasar & $4.8 \%$ & $4.5 \%$ & $4.7 \%$ & \\
\hline & Defender & $20.8 \%$ & $30.3 \%$ & $25.6 \%$ & \\
\hline & Otros & $7.5 \%$ & $9.2 \%$ & $8.4 \%$ & \\
\hline \multirow{5}{*}{ Acción técnico-táctica individual en entrenamiento } & Lanzar & $50.8 \%$ & $40.5 \%$ & $45.6 \%$ & \multirow{5}{*}{$\begin{array}{c}.004^{* *} \\
\Phi=.069\end{array}$} \\
\hline & Fintar & $17.7 \%$ & $19 \%$ & $18.3 \%$ & \\
\hline & Pasar & $5.1 \%$ & $3.6 \%$ & $4.3 \%$ & \\
\hline & Defender & $19.1 \%$ & $24.2 \%$ & $21.7 \%$ & \\
\hline & Otros & $7.2 \%$ & $12.8 \%$ & $10.1 \%$ & \\
\hline \multirow{2}{*}{ Preferencia del jugador por atacar o por defender } & Atacar & $71.7 \%$ & $62.9 \%$ & $67.3 \%$ & \multirow{2}{*}{$\begin{array}{c}.013^{* *} \\
\Phi=.058\end{array}$} \\
\hline & Defender & $28.3 \%$ & $37.1 \%$ & $32.7 \%$ & \\
\hline
\end{tabular}

**Variables con un nivel de significación $\rho<.05$.

El análisis de la preferencia de realización de acciones técnico-tácticas individuales muestra diferencias estadísticamente significativas tanto en las acciones técnico-tácticas en partido $\left(\chi 2(5, \mathrm{~N}=837)=15.031_{\mathrm{a}}, \mathrm{p}=.010\right)$, como en la preferencia de acciones técnico-tácticas en entrenamiento $(\chi 2(5, \mathrm{~N}=835)=$ $17.060, \mathrm{p}=.004)$, siendo en ambos casos la acción preferida tanto por los chicos como por las chicas el lanzar a portería.

En cuanto a la preferencia de atacar frente a defender, los resultados muestran también diferencias estadísticamente significativas $(\chi 2(1, \mathrm{~N}=740)=6.547 \mathrm{~b}, \mathrm{p}=.013)$, mostrándose en ambos sexos una mayor preferencia por el atacar frente al defender.

Preferencias por la fase de juego y las acciones técnico-tácticas

La tabla 2 analiza las preferencias de los jugadores por las fases del juego, la opinión que creen que tienen sus entrenadores sobre atacar y defender, y la preferencia en acciones técnico-tácticas. 
Tabla 2. Preferencias en fases de juego y en acciones técnico-tácticas*.

\begin{tabular}{lccccccc}
\hline \multirow{2}{*}{ Variable } & \multicolumn{2}{c}{ Masculino } & \multicolumn{2}{c}{ Femenino } & \multicolumn{3}{c}{ Total } \\
\cline { 2 - 6 } & Media & DT & Media & DT & Media & DT & $\rho$ valor \\
\hline ¿Cuánto te gusta atacar? & 9.02 & 1.279 & 8.63 & 1.303 & 8.82 & 1.305 & $.000^{* *}$ \\
¿Cuánto te gusta defender? & 8.37 & 1.670 & 8.42 & 1.528 & 8.39 & 1.599 & .982 \\
¿Crees que a tu entrenador le gusta más atacar? & 7.99 & 1.524 & 8 & 1.656 & 7.99 & 1.590 & .653 \\
¿Crees que a tu entrenador le gusta más defender? & 9.14 & 1.410 & 8.79 & 1.635 & 8.96 & 1.535 & $.001^{* *}$ \\
¿Cuánto te gusta lanzar desde7 metros indiferentemente del resultado? & 7.58 & 1.979 & 6.98 & 2.296 & 7.28 & 2.163 & $.000^{* *}$ \\
¿Cuánto te gusta lanzar desde6 metros indiferentemente del resultado? & 8.4 & 1.757 & 8.19 & 1.893 & 8.3 & 1.828 & .120 \\
¿Cuánto te gusta lanzar desde 9 metros indiferentemente del resultado? & 7.35 & 2.267 & 6.76 & 2.401 & 7.06 & 2.352 & $.000^{* *}$ \\
¿Cuánto te gusta lanzar desde 7 metros y conseguir gol? & 8.65 & 1.711 & 8.25 & 2.130 & 8.45 & 1.941 & $.021^{* *}$ \\
¿Cuánto te gusta lanzar desde 6 metros y conseguir gol? & 8.89 & 1.418 & 8.63 & 1.785 & 8.76 & 1.617 & .082 \\
¿Cuánto te gusta lanzar desde 9 metros y conseguir gol? & 8.76 & 1.963 & 8.18 & 2.427 & 8.47 & 2.227 & $.000^{* *}$ \\
¿Cuánto te gusta coger rechaces en defensa? & 8.07 & 1.868 & 8.23 & 1.904 & 8.15 & 1.887 & .105 \\
¿Cuánto te gusta coger rechaces en ataque? & 7.84 & 1.946 & 7.96 & 1.916 & 7.9 & 1.931 & .381 \\
¿Cuánto te gusta provocar golpes francos a los jugadores? & 7.25 & 2.310 & 6.79 & 2.547 & 7.02 & 2.441 & $.010^{* *}$ \\
¿Cuánto te gusta interceptar pases? & 8.66 & 1.581 & 8.34 & 1.732 & 8.5 & 1.665 & $.008^{* *}$ \\
¿Cuánto te gusta recuperar balones? & 8.73 & 1.554 & 8.55 & 1.713 & 8.64 & 1.637 & .167 \\
¿Cuánto te gusta provocar faltas en ataque? & 7.9 & 2.708 & 6.74 & 3.195 & 7.32 & 3.016 & $.000^{* *}$ \\
¿Cuánto te gusta dar asistencias? & 8.13 & 1.670 & 7.92 & 2.063 & 8.02 & 1.877 & .564 \\
¿Cuánto te gusta realizar fintas? & 8.33 & 1.799 & 8.14 & 1.820 & 8.23 & 1.811 & .067 \\
¿Cuánto te gusta realizar buenas defensas? & 9.02 & 1.362 & 8.82 & 1.592 & 8.92 & 1.485 & .185 \\
¿Cuánto te gusta tocar el balón durante el juego de ataque? & 7.9 & 2.188 & 8.34 & 1.794 & 8.12 & 2.011 & $.009^{* *}$ \\
\hline
\end{tabular}

${ }^{*}$ La unidad de medida es una escala Likert en la que 0 es nada y 10 totalmente.

${ }^{* *}$ Variables con un nivel de significación $\rho<.05$.

Los resultados de la tabla 2 muestran que atacar es la fase del juego preferida tanto por los chicos $(\mathrm{x}=9,02)$ como por las chicas $(\bar{x}=8,63)$, encontrándose diferencias estadísticamente significativas entre los grupos en la preferencia por atacar $(\mathrm{z}=$ $-5.110, \rho=.000)$.

En el mismo sentido, tanto los chicos $(\mathrm{x}=9,14)$ como las chicas $(\bar{x}=8,79)$ tienen la creencia de que lo que más les gusta a sus entrenadores es defender, encontrándose diferencias estadísticamente significativas entre grupos $(z=-3.403, \rho=$ $.001)$.

Por el contrario, el análisis de la variable sobre "cuánto crees que le gusta a tu entrenador atacar" no muestra diferencias estadísticamente significativas ( $\mathrm{z}=-.449, \rho=.653$ ).

En cuanto a la preferencia por las diferentes acciones técnico-tácticas los resultados muestran diferencias en varias de ellas. En la variable “¿Cuánto te gusta lanzar desde 7 metros indiferentemente del resultado?" se encuentran diferencias estadísticamente significativas entre ambos grupos $(\mathrm{z}=-3.747$, $\rho=.000)$, siendo esta acción preferida en mayor medida por los chicos $(\overline{\mathrm{x}}=7,58)$, que por las chicas $(\overline{\mathrm{x}}=6,98)$.

Los resultados sobre la variable "¿Cuánto te gusta lanzar desde 9 metros indiferentemente del resultado?” muestra también diferencias estadísticamente significativas $(\mathrm{z}=-3.675$, $\rho=.000)$, siendo en este caso mayor la preferencia de los chi$\cos (\overline{\mathrm{x}}=7,35)$, frente a las chicas $(\overline{\mathrm{x}}=6,76)$.

El análisis de la variable “¿Cuánto te gusta lanzar desde 7 metros y conseguir gol?” también muestra diferencias significativas entre los grupos $(\mathrm{z}=-2.307, \rho=.021)$, mostrando una mayor preferencia los chicos $(\overline{\mathrm{x}}=8,65)$, que las chicas $(\overline{\mathrm{x}}=8,25)$.

En cuanto a la variable "¿Cuánto te gusta lanzar desde 9 metros y conseguir gol?” las diferencias encontradas también son estadísticamente significativas $(\mathrm{z}=-3.585, \rho=.000)$, con una mayor preferencia de los chicos $(\overline{\mathrm{x}}=8,76)$, frente a las chicas $(\overline{\mathrm{x}}=8,18)$.

Los resultados del análisis estadístico sobre la variable "¿Cuánto te gusta provocar golpes francos a los jugadores?" muestra diferencias estadísticamente significativas $(\mathrm{z}=$ -2.567, $\rho=.010)$, encontrándose más satisfacción en los chi$\cos (\overline{\mathrm{x}}=7,25)$, que en las chicas $(\overline{\mathrm{x}}=6,79)$ al realizar esta acción técnico-táctica.

En cuanto al análisis de la variable "¿Cuánto te gusta interceptar pases?” también se encuentran diferencias significativas $(\mathrm{z}=-2.653, \rho=.008)$, siendo mayor la preferencia de los chicos $(\overline{\mathrm{x}}=8,66)$, que la de las chicas $(\overline{\mathrm{x}}=8,34)$. 
Los resultados de la variable “¿Cuánto te gusta provocar faltas en ataque?" muestran diferencias significativas ( $\mathrm{z}=$ -5.986, $\rho=.000)$, con una mayor preferencia por los chicos $(\overline{\mathrm{x}}=7,9)$, que por las chicas $(\overline{\mathrm{x}}=6,74)$.

Finalmente, el análisis de la variable “¿Cuánto te gusta tocar el balón durante el juego de ataque?”, referido al tiempo de posesión individual del balón durante la fase ofensiva del juego,también muestra diferencias estadísticamente significativas $(\mathrm{z}=-2.626, \rho=.009)$, aunque en este caso existe una ma- yor preferencia por las chicas en realizar esta acción $(\overline{\mathrm{x}}=8,34)$ que por los chicos $(\overline{\mathrm{x}}=7,9)$.

\section{Preferencias por las situaciones de igualdad y desigualdad numérica de juego}

La tabla 3 analiza las preferencias que tienen los jugadores de balonmano según su rendimiento deportivo por las situaciones de igualdad o desigualdad numérica de juego en las que participan.

Tabla 3. Preferencias en cuanto a situaciones de igualdad o desigualdad numérica de juego*.

\begin{tabular}{|c|c|c|c|c|c|c|c|}
\hline \multirow{2}{*}{ Variable } & \multicolumn{2}{|c|}{ Masculino } & \multicolumn{2}{|c|}{ Femenino } & \multicolumn{2}{|l|}{ Total } & \multirow{2}{*}{ - $\rho$ valor } \\
\hline & Media & DT & Media & DT & Media & DT & \\
\hline $1 \mathrm{x} 0$ & 6.77 & 3.334 & 7.9 & 2.788 & 7.34 & 3.124 & $.000^{* *}$ \\
\hline $1 \times$ portero & 8.54 & 1.928 & 8.6 & 1.905 & 8.57 & 1.915 & .543 \\
\hline $1 \times 1$ & 8.13 & 1.745 & 7.67 & 1.988 & 7.9 & 1.884 & $.001^{* *}$ \\
\hline $2 \times 1$ & 8.1 & 1.852 & 8.01 & 1.986 & 8.05 & 1.920 & .601 \\
\hline $2 \times 2$ & 8.15 & 1.581 & 7.87 & 1.776 & 8.01 & 1.686 & $.046^{* *}$ \\
\hline $3 \times 3$ & 8.16 & 1.617 & 7.94 & 1.788 & 8.05 & 1.707 & .095 \\
\hline Otras & 8.7 & 1.415 & 8.6 & 1.607 & 8.65 & 1.513 & .660 \\
\hline
\end{tabular}

*La unidad de medida es una escala Likert en la que 0 es nada y 10 totalmente.

**Variables con un nivel de significación $\rho<.05$.

El análisis de la preferencia por situaciones de juego muestra que la situación de igualdad o desigualdad numérica que más gusta realizar es la de " 1 x portero", tanto en chicos $(\bar{x}=8,54)$, como en chicas ( $\mathrm{x}=8,6)$; así como "otro tipo de situaciones de igualdad o desigualdad numérica”, entendiendo estas situaciones como cualquier otra situación de igualdad o desigualdad numérica de juego que no se ha presentado previamente en el análisis de la tabla, tanto en chicos $(\overline{\mathrm{x}}=8,7)$, como en chicas $(\overline{\mathrm{x}}=8,6)$.

Los resultados muestran diferencias estadísticamente significativas en las situaciones de juego de " 1 x 0 ", es decir situaciones en las que el jugador lanza sin oposición defensiva ni portero contra portería, $(\mathrm{z}=-4.960, \rho=.000)$, existiendo una mayor preferencia por las chicas $(\overline{\mathrm{x}}=7,9)$ que por los chicos $(\bar{x}=6,77)$; también se encuentran diferencias en las situaciones de "1 x 1", $(\mathrm{z}=-3.361, \rho=.001)$, con una mayor preferencia por parte de los chicos ( $\overline{\mathrm{x}}=8,13)$, que de las chicas $(\overline{\mathrm{x}}=7,67)$; por último, en las situaciones de " 2 x 2 " también se encuentran diferencias estadísticamente significativas, $(\mathrm{z}=-1.996$, $\overline{\mathrm{x}}=.046$ ), existiendo una mayor preferencia por los chicos $(\overline{\mathrm{x}}=8,15)$, que por las chicas $(\overline{\mathrm{x}}=7,87)$.

\section{Discusión}

Una vez analizados los resultados del presente trabajo, estos muestran que la acción por la que se muestra mayor preferencia es la de lanzar a portería tanto en los chicos como en las chicas, ya sea en entrenamiento o en competición. Siendo además esta preferencia mayor en el género masculino (50,5\% en partido y $50,8 \%$ en entrenamiento), que en el femenino (38,5\% en partido y $40,5 \%$ en entrenamiento).

Además, de todos los tipos de lanzamientos posibles analizados el que muestra una mayor preferencia tanto en chicos $(\bar{x}=8,89 \pm 1.41)$, como en chicas $(\bar{x}=8,63 \pm 1.78)$, es la acción de lanzar y conseguir gol desde 6 metros.

Estos datos concuerdan con los resultados encontrados por otros estudios, que también encuentran la acción de lanzar como la favorita por los jugadores, ya sea en estudios centrados en el balonmano (García-Angulo et al., 2014; Ortega et al., 2015) o en otros deportes como el baloncesto (Ortega et al., 2009; Palao, Ortega, y Olmedilla, 2007). Así como con las actuales propuestas teóricas, que confieren a la acción de lanzar en particular, y a la fase ofensiva en general, un elemento esencial dentro del proceso formativo de los jóvenes deportista, (American Sport Education Program, 2001; Antón, 1998; Palao et al., 2007).

En cuanto a la preferencia que muestran los jugadores por atacar que por defender, se muestra que las chicas tienen una mayor preferencia por defender que los chicos. Esto es quizá debido a que las motivaciones que tienen los chicos y las chicas a la hora de practicar deporte son diferentes, ya que mientras las chicas muestran una mayor orientación a la tarea, en la que se valoran los progresos a nivel individual, los chicos tienen una mayor orientación hacia el ego en el que la motiva- 
ción por la práctica deportiva se basa en el rendimiento en sí (Cervelló et al., 2007; Santos-Rosa et al., 2007).

Del mismo modo, si se analizan las acciones técnico-tácticas de una manera concreta, la acción técnico-táctica que más satisfacción ocasiona a los jóvenes deportistas es la de realizar buenas defensas, tanto en los chicos ( $\mathrm{x}=9,02 \pm 1.36)$, como en las chicas $(\overline{\mathrm{x}}=8,82 \pm 1.59)$; obteniendo también una puntuación muy alta otras acciones defensivas como interceptar pases $(\overline{\mathrm{x}}=8,66 ; \overline{\mathrm{x}}=8,34)$, o recuperar balones $(\overline{\mathrm{x}}=8,73 ; \overline{\mathrm{x}}=8,55)$.

Estos datos son similares a los encontrados por otros estudios tanto en balonmano (García-Angulo et al., 2014; Ortega et al., 2015) como en el baloncesto (Ortega et al., 2009; Palao et al., 2007). Este hecho muestra que a pesar de que la fase preferida del juego es la ofensiva y que los actuales modelos defienden el basar el entrenamiento en el ataque sobre la defensa, la realización de una buena defensa incide positivamente en la satisfacción del jugador en proceso formativo.

Modelos como el Teaching Games for Understanding (Bunker y Thorpe, 1982; Thorpe y Bunker, 1989), el Sport Education (Siedentop, 2002; Siedentop, Hastie, y van der Mars, 2004) o el modelo de Enseñanza Comprensiva (Cárdenas, 2003; Castejón, 2010) defienden una enseñanza basada en los aspectos tácticos, en los que debe de primar la enseñanza basada en el ataque sobre la defensa en base a que así se logrará una mayor participación por parte de los jóvenes deportistas, siendo éste un elemento fundamental para lograr una mayor satisfacción y en consecuencia una mayor adherencia a la práctica deportiva (Cárdenas, 2003; Ortega, 2010).

Un dato muy a tener en cuenta es que los jóvenes jugadores muestran una alta preferencia por la realización de ciertas acciones de carácter defensivo, este dato concuerda con lo encontrado en otros estudios de balonmano (García-Angulo et al., 2014; Ortega et al., 2015) y en baloncesto (Ortega et al., 2009; Palao et al., 2007) y puede estar relacionado con lo que creen que les solicita su entrenador en partidos y entrenamientos y en lo que creen más adecuado según la visión del mismo (Ortega, 2010).

Muy relacionado con lo anterior están los resultados encontrados al preguntar a los jóvenes jugadores sobre qué creen que les gusta más a sus entrenadores si atacar o defender, ya que ambos grupos respondieron que la defensa $(\bar{x}=9,14 \pm 1.41$; $\overline{\mathrm{x}}=8,79 \pm 1.63)$.

Este hecho sin duda influenció a la mayor preferencia por ciertas acciones defensivas a ambos grupos, ya que el entrenador es concebido en estas edades tan tempranas como una figura de autoridad y un líder dentro del grupo (Fenoy y Campoy, 2012; Rosado, Palma, Mesquita, y Moreno, 2007; Ruiz, 2007).

$\mathrm{Al}$ analizar las acciones técnico-tácticas de una manera específica se encuentran diferencias estadísticamente significativas entre el grupo de los chicos y el grupo de las chicas.

Concretamente se encuentran diferencias en la realización de varias acciones de lanzamiento independientemente del resultado, como son: “'Cuánto te gusta lanzar de 7 metros indiferentemente del resultado?” “¿Cuánto te gusta lanzar de 9 metros indiferentemente del resultado?", en ambas existe una mayor preferencia por parte de los chicos que de las chicas $(\bar{x}=7,58 ; \bar{x}=6,98$ y $\bar{x}=7,35 ; \bar{x}=6,76)$.

Este hecho concuerda con lo hallado en otros estudios y en cierta medida está relacionado con la percepción eficacia y la satisfacción, ya que los chicos al tener mayor nivel físico en estas edades pueden tener una mayor percepción de la eficacia al lanzar, frente al grupo de las chicas (García-Angulo et al., 2014; Leo, García, Parejo, Sánchez, y García-Mas, 2009; Myers, Feltz, y Short, 2004; Ortega et al., 2015; Watson, Chemers, y Preiser, 2001).

En el mismo sentido van las variables “¿Cuánto te gusta lanzar desde 7 metros y conseguir gol?” y “Cuánto te gusta lanzar desde 9 metros y conseguir gol?” ya que los chicos prefieren en mayor medida las acciones en las que se lanza con eficacia que las chicas ( $\bar{x}=8,65 ; \bar{x}=8,25$ y $\bar{x}=8,76 ; \bar{x}=8,18)$. Estos resultados coinciden con los encontrados en otros trabajos, y se fundamenta en que en líneas generales las chicas tienen una mayor orientación hacia la tarea, interesándose por factores que van más allá del rendimiento deportivo, mientras que los chicos tienden a una orientación de la motivación al ego (Castillo et al., 2000; Cecchini et al., 2008; Gano-Overway y Duda, 2001; Hanrahan y Biddle, 2002; Hanrahan y Cerin, 2009; Kavussanu y Roberts, 2001).

Del mismo modo en las variables que se refieren a ¿Cuánto te gusta provocar golpes francos a los jugadores?” a “Cuánto te gusta interceptar pases?” y a “¿Cuánto te gusta provocar faltas en ataque?" se encuentran resultados similares, ya que los chicos tienen una mayor preferencia por la realización de acciones defensivas u ofensivas en las que obtengan una recompensa más individual que grupal, es decir, el hecho de provocar un golpe franco o provocar una falta responde a la mayor orientación hacia el ego por parte de los chicos, ya que supone una acción en la que reciben un reconocimiento de un logro individual que contribuye al equipo, pero que en definitiva es un logro individual que afianza esa orientación hacia el ego (Cecchini et al., 2008; Cervelló et al., 2007; Santos-Rosa et al., 2007).

Por el contrario, el análisis de la preferencia por tocar el balón en la fase de ataque muestra una mayor preferencia de las chicas frente a los chicos, esto se debe a los mismos motivos que las variables técnico-tácticas anteriores, ya que las chicas muestran una mayor orientación a la tarea en la que la participación deportiva colectiva es por sí misma un hecho motivante y en la que la motivación no se basa meramente en aspectos de rendimiento, sino en otros factores como el concepto de equipo, entendido como una colectividad; por el contrario los chicos muestran una mayor preferencia por las acciones que supongan rendimiento deportivo, es decir una 
mayor orientación hacia el ego (Cervelló et al., 2007; Hanrahan y Cerin, 2009).

Finalmente el hecho de que las chicas prefieran en mayor medida las situaciones de " 1 x 0 " frente a los chicos coincide con lo expuesto en otros trabajos, con la mayor percepción de eficacia por parte de los chicos, y a que los chicos prefieren en mayor medida las situaciones de competición reales, ya que éstas se adecúan a la mayor orientación hacia el ego, mientras que la acción de " 1 x 0 ” se adapta más a la orientación hacia la tarea, ya que permite mejorar el lanzamiento sin la necesidad de competir contra nadie, además de que por sí misma se realiza continuamente la acción de lanzar que es la que más motivación ocasiona (Cervelló et al., 2007; García-Angulo et al., 2014; Ortega et al., 2015; 2009; Palao et al., 2007).

El hecho de que los chicos prefieran las situaciones de igualdad numérica " 1 x 1 ” y “2 x 2" se debe fundamentalmente a dos motivos: a) a que este tipos de situaciones numéricas son las que más se parecen a la realidad competitiva, y por tanto más se relacionan con la orientación al ego; y b) a que quizá el mayor grado de desarrollo físico de los chicos en estas edades haga que prefieran situaciones técnico-tácticas más complejas que producen en su caso una mayor satisfacción (Cárdenas, 2003; Cervelló y col., 2007; García-Angulo et al., 2014; Ortega, 2010; Ortega et al., 2015).

\section{Conclusiones}

Una vez finalizado el estudio se extraen una serie de conclusiones que pueden servir para mejorar la orientación del entrenamiento en balonmano para chicos y chicas en etapas de formación:

a) La acción técnico-táctica favorita tanto en chicos como en chicas es la de lanzar a portería, dato que coincide con lo encontrado en otros trabajos sobre balonmano y sobre otros deportes colectivos en etapas de formación.

b) La fase del juego favorita en ambos grupos es la de atacar, algo que coincide con la idea de los actuales modelos teóricos de entrenar más en ataque que en defensa.

c) Aun prefiriendo en mayor medida el ataque, las chicas muestran una mayor preferencia por la defensa que los chicos, este hecho ha sido relacionado en diversos estu- dios con una mayor orientación a la tarea de las chicas frente a una orientación al ego de los chicos.

d) A pesar de ser la fase de ataque la favorita por ambos grupos, ambos grupos muestran una alta preferencia en la realización de ciertas acciones defensivas.

e) El análisis de las situaciones de igualdad o desigualdad numérica de juego arroja que las chicas prefieren en mayor medida las situaciones de " 1 x 0 ", mientras que los chicos las situaciones de igualdad numérica "1 x 1 " y “2 x 2", quizá debido al mayor carácter competitivo de éstas últimas y al mayor acercamiento a la realidad competitiva.

Estas conclusiones pueden servir a los entrenadores para optimizar los procesos de enseńanza-aprendizaje del balonmano, entendiendo que las preferencias técnico-tácticas no son las mismas en chicos y chicas y que el entrenamiento debe de adaptarse a sus preferencias con el fin de conseguir una mayor adherencia a la práctica.

En este sentido, los técnicos deportivos deben de diseñar unas tareas de entrenamiento en las que prime el trabajo ofensivo sobre el defensivo, y en donde se produzcan el mayor número posible de lanzamientos y situaciones de uno contra portero en ambos sexos. Sin embargo, se debe destacar que el plantear tareas en donde se consiga realizar acciones defensivas con eficacia, también garantizará satisfacción en los jóvenes deportistas.

De igual forma, es necesarios eguir profundizando en estos aspectos en general, y en sus posibles causas en particular, analizando de manera más precisa la relación entre estos datos y las teorías motivacionales actuales.

Como puntos fuertes del estudio, cabría destacar que es necesario conocer las prioridades preferencias y puntos de vista de los jugadores, para a partir de ellos, poder establecer progresiones deportivas que se ajusten a sus demandas. En el presente estudio, se presentan dichos datos separados por género, en una muestra muy importante cuantitativamente.

Como puntos débiles, cabe destacar que los datos proceden de jugadores federados, pero de elevado nivel (participantes en Campeonato de España), lo que no es del todo exportable a otras poblaciones que practican balonmano en entornos más recreativos o con un nivel menor de competitividad.

\section{Bibliografía}

1. Alonso, N., Martínez-Galindo, C., Moreno, J.A. \& Cervelló, E. (2005). Relación del género del alumno y el tipo de centro con la motivación, disciplina, trato de igualdad y estado de flow en Educación Física. In A. Díaz (Ed.), V Congreso Internacional de Educación Física e Interculturalidad. Murcia, España: Instituto de Ciencias del Deporte.

2. American Sport Education Program. (2001). Coaching youth basketball (3rd ed.). Champaign, IL: Human Kinetics.

3. Antón, J.L. (1998). Balonmano: táctica grupal ofensiva: concepto, estructura y metodología. Madrid: Gymnos.
4. Balaguer, I., Castillo, I.\& Duda, J.L. (2007). Propiedades psicométricas de la Escala de Motivación Deportiva en deportistas espańoles. Revista Mexicana de Psicología, 24, 197-207.

5. Balaguer, I., Castillo, I.\& Duda, J.L. (2008). Apoyo a la autonomía, satisfacción de las necesidades, motivación y bienestar en deportistas de competición: un análisis de la teoría de la autodeterminación. Revista de Psicología del Deporte, 17, 123-139.

6. Borrego, F.J., Carrillo, A.B.\& Díaz, A. (2015). Análisis descriptivo de la práctica físico-deportiva e intereses de práctica en escolares de $5^{\circ}$ y $6^{\circ}$ de primaria. SporTK, 4(1), 23-28. 
7. Bunker, D.\& Thorpe, R. (1982). A model for the teaching of games in the secondary school. Bulletin of Physical Education, 10:9-16.

8. Cárdenas, D. (2003). El proceso de formación táctica colectiva desde una perspectiva constructivista. En A. López, C. Jiménez y R. Aguado (Eds.), Didáctica del baloncesto en las etapas de formación (pp.179-209). Madrid: Editores.

9. Castejón, F.J. (2010). Deporte y enseñanza comprensiva. Sevilla: Wanceulen.

10. Castillo, I., Balaguer, I.\& Duda, J.L. (2000). Las orientaciones de meta y los motivos de práctica deportiva en los jóvenes deportistas valencianos escolarizados. Revista de Psicología del Deporte, 9, 37-50.

11. Cecchini, J.A., González, C.\& Montero, J. (2008). Participación en el deporte. Orientación de metas y funcionamiento moral. Revista Latinoamericana de Psicología, 40(3), 497-509.

12. Cervelló, E., Escartí, A.\& Guzmán, F.J. (2007). Youth sport dropout from the achievement goal theory. Psicothema, 19(1), 65-71.

13. Chantal, Y., Guay, F., Dobreva-Martinova, T. \& Vallerand, R.J. (1996). Motivation and elite perfomance: An exploratory investigation with Bulgarian athletes. International Journal of Sport Psychology, 27, 173-182.

14. Chen, A. (2001). A theoretical conceptualization for motivation research in physical education: An integrated perspective. Quest, 53, 35-58.

15. Deci, E.L. \& Ryan, R.M. (1985). Intrinsic motivation and self-determination in human behaviour. New York: Plenum.

16. Deci, E.L. \& Ryan, R.M. (2000). The "what" and "why" of goal pursuits: Human needs and the self-determination of behaviour. Psychologicalinquiry, 11, 227-268.

17. Fenoy, J.\& Campoy, L. (2012). Rendimiento deportivo, estilos de liderazgo y evitación experiencial en jóvenes futbolistas almerienses. Revista de Psicología del Deporte, 21(1), 137-142.

18. Gano-Overway, L.A. \& Duda, J.L. (2001). Personal theories of achievement motivation among African and White Mainstream American athletes. International Journal of Sport Psychology, 32, 335-354

19. García-Angulo, A., Ortega, E.\& Mendoza, R. (2014). Grado de satisfacción y preferencias de jugadores de balonmano en acciones técnico-tácticas según la categoría de juego. E-balonmano.com: Revista de Ciencias del Deporte 10(3), 139-148.

20. Giménez, F.J.\& Sáenz-López, P. (2006). Planificación del entrenamiento. En C. Torres (Eds.), La formación del educador deportivo en baloncesto: Bloque especifico nivel II (pp.79-102). Sevilla: Wanceulen Editorial Deportiva S.L.

21. Giménez, F.J.\& Sáenz-López, P. (2007). Estrategias lúdicas para mejorar la percepción y la decisión de los jugadores y jugadoras. En A.C. Jiménez y G. Ortega (Coord), Baloncesto en la Iniciación (pp.123-138). Sevilla: Wanceulen Editorial Deportiva S.L.

22. González, A., Donolo, D., Rinaudo, C.\& Paoloni, V. (2011). Relaciones entre motivación, emoción y rendimiento académico en universitarios. Estudios de psicología, 32(2), 257-270.

23. González, J.\& Martínez, J.M. (2015). Estrategias de afrontamiento y personalidad en la adaptation de jóvenes a su práctica deportiva. Diferencias de género. SporTK, 4(1), 57-62.

24. Hanrahan, S.J. \& Biddle. S.J.H. (2002). Measurement of achievement orientations: Psychometric measures, gender and sport differences. European Journal of Sport Science, 2(5), 1-12.

25. Hanrahan, S.J. \& Cerin, E. (2009). Gender, level of participation and type of sport: differences in achievement goal orientation and attributional style. Journal of Science and Medicine in Sport, 12, 508-512.

26. Kavussanu, M.\& Roberts, G.C. (2001). Motivation in physical activity contexts: The relationship of perceived motivational climate to intrinsic motivation and self-efficacy. Journal of Sport and Exercise Psychology, 18, 264-280.

27. Leo, F.M., García, T., Parejo, I., Sánchez, P.A.\& García-Mas, A. (2009). Aplicación de un programa de intervención para la mejora de la cohesión y la eficacia en jugadores de baloncesto. Cuadernos de Psicología del deporte, 9(1), 73-84.
28. Martínez-Galindo, C., Alonso, N., Cervelló, E.\& Moreno, J. A. (2009). Perfiles motivacionales y disciplina en clases de educación física. Diferencias según las razones del alumnado para ser disciplinado y la percepción del trato generado por el profesorado en el aula. Cultura y Educación, 21, 331-343.

29. Moreno, J. A., Hellín, P., Hellín, G. \& Cervelló, E. (2006). Efectos del género, la edad y la práctica físico-deportiva en las estrategias de disciplina, la orientación disposicional y la motivación autodeterminada en estudiantes adolescentes de Educación Física. In A. Díaz (Ed.), VI Congreso Internacional de Educación Física e Interculturalidad. Murcia, España: Instituto de Ciencias del Deporte.

30. Myers, N.D., Feltz, D.L.\& Short, S.E. (2004). Collective efficacy and team performance: A longitudinal study of collegiate football teams. Group Dynamics: Theory, Research, and Practice, 8:126-138

31. Nicholls, J.G. (1989). The competitive ethos and democratic education Cambridge. MASS: Harvard University Press.

32. Ortega, E. (2010). Medios técnico-tácticos colectivos en baloncesto en categorías de formación. Revista Internacional de Medicina y Ciencias de la Actividad Física y el Deporte, 10(38), 234-244.

33. Ortega, E., García-Angulo, A., Mendoza, R.\& López, J.M. (2015). Grado de satisfacción y preferencias de jugadores de balonmano en acciones técnico-tácticas según su rendimiento deportivo. Journal of Sport and Health Research. 7(3), 203-214.

34. Ortega, E., Giménez, J.M., Palao, J.M. \& Sainz de Baranda, P. (2008) Diseño y validación de un cuestionario para valorar las preferencias y satisfacciones en jóvenes jugadores de baloncesto. Cuadernos de Psicología del Deporte, 8(2), 39-58.

35. Ortega, E., Palao, J.M., Sainz de Baranda, P.\& García, L.M. (2009) Preferences and levels of satisfaction in technical and tactical actions and in type of offense and defense utilized in competition by youth basketball players. Revista de Psicología del Deporte, 18(3), 343-348.

36. Ortega, E., Piñar, M. I., \& Cárdenas, D. (1999). El estilo de juego de los equipos de baloncesto en las etapas de formación. Granada: Editores

37. Palao, J.M., Ortega, E.\& Olmedilla, A. (2007). Technical and tactical preferences basketball players in formative years. Iberian Congress on Basketball Research, 4:38-41.

38. Petherick, C.\& Weigand, D. (2002). The relationship of dispositional goal orientations and perceived motivational climates on indices of motivation in male and female swimmers. International Journal of Sport Psychology, 33, 218-237.

39. Rosado, A., Palma, N., Mesquita, I. \& Moreno, P. (2007). Percepción de los jugadores de fútbol, de distinto nivel sobre sus entrenadores. Revista de Psicología del Deporte, 16(2), 151-165.

40. Ruiz, R. (2007). Características de liderazgo en el deporte del judo. Revista de Psicología del Deporte 16(1), 9-24.

41. Santos-Rosa, F.J., García, T., Jiménez, R., Moya, M. \& Cervelló, E. (2007). Predicción de la satisfacción con el rendimiento deportivo en jugadores de tenis: efecto de las claves situacionales. Motricidad: revista de ciencias de la actividad física y del deporte, 18, 41-60.

42. Siedentop, D. (2002). Sport Education: a retrospective. Journal of Teaching and Physical Education, 21:409-418.

43. Siedentop, D., Hastie, P.A. \& van der Mars, H. (2004). Complete guide to Sport Education. Champaign, IL: Human Kinetics.

44. Thorpe, R.\& Bunker, D. (1989). A changing focus in games education In L. Almond (Ed.), The place of physical education in schools (pp. 42-71). London: Kogan Page.

45. Treasure, D.C.\& Roberts, G.C. (2001). Students' perceptions of the motivational climate, achievement beliefs and satisfaction in physical education. Research Quarterly of Exercise and Sport, 72, 165-175.

46. Watson, C.B., Chemers, M.M. \& Preiser, N. (2001). Collective efficacy: A multilevel analysis. Personality and Social Psychology Bulletin, 27:1057-1068. 
ANEXO I.

\section{CUESTIONARIO PARA JÓVENES JUGADORES/AS DE BALONMANO}

El presente cuestionario forma parte de un trabajo de investigación que se está llevando a cabo por profesores de la Universidad de Murcia El estudio pretende evaluar las características y circunstancias que rodean a los jóvenes que se inician en el BALONMANO. El cuestionario es anónimo, te pedimos por favor que seas sincero en tus respuestas. Para cumplimentarlo, marca las respuestas que consideres más adecuadas en cada pregunta, teniendo en cuenta que, excepto cuando se indique, solo deberás marcar una respuesta. Cuando sea necesario, rellena las líneas con letra clara.

COMUNIDAD: FECHA DE NACIMIENTO: GENERO: M F

CATEGORÍA: ALTURA: PESO: .

No AÑOS ENTRENANDO: No DÍAS A LA SEMANA: HORAS DE ENTRENAMIENTO POR SEMANA:

Puesto específico: en tus inicios, actualmente y en el que más te gustaría jugar (marca con una X)

\begin{tabular}{|c|c|c|c|c|c|}
\hline & Central & Lateral & Extremo & Pivote & Portero \\
\hline \multicolumn{6}{|l|}{ En tus inicios } \\
\hline \multicolumn{6}{|l|}{ Actualmente } \\
\hline Él que más te gustaría & & & & & \\
\hline
\end{tabular}

SEÑALA, RODEANDO CON UN CÍRCULO DE 0 (NADA) A 10 (MUCHO) EL GRADO DE SATISFACCIÓN QUE TIENES CUANDO REALIZAS CADA UNA DE LAS SIGUIENTES ACCIONES DEL JUEGO:

\begin{tabular}{|c|c|}
\hline ACCIÓN DE JUEGO & GRADO DE SATISFACCIÓN \\
\hline Lanzamiento de 7 metros indiferentemente del resultado & $0-1-2-3-4-5-6-7-8-9-10$ \\
\hline Lanzamiento de 6 metros indiferentemente del resultado & $0-1-2-3-4-5-6-7-8-9-10$ \\
\hline Lanzamiento de 9 metros indiferentemente del resultado & $0-1-2-3-4-5-6-7-8-9-10$ \\
\hline Lanzamiento de 7 metros anotado & $0-1-2-3-4-5-6-7-8-9-10$ \\
\hline Lanzamiento de 6 metros anotado & $0-1-2-3-4-5-6-7-8-9-10$ \\
\hline Lanzamiento de 9 metros anotado & $0-1-2-3-4-5-6-7-8-9-10$ \\
\hline Coger rechaces en defensa & $0-1-2-3-4-5-6-7-8-9-10$ \\
\hline Coger rechaces en ataque & $0-1-2-3-4-5-6-7-8-9-10$ \\
\hline Provocar golpes francos a los defensores & $0-1-2-3-4-5-6-7-8-9-10$ \\
\hline Interceptar un pase & $0-1-2-3-4-5-6-7-8-9-10$ \\
\hline Recuperar balones & $0-1-2-3-4-5-6-7-8-9-10$ \\
\hline Provocar una falta en ataque & $0-1-2-3-4-5-6-7-8-9-10$ \\
\hline Dar una asistencia & $0-1-2-3-4-5-6-7-8-9-10$ \\
\hline Realizar fintas & $0-1-2-3-4-5-6-7-8-9-10$ \\
\hline Realizar una buena defensa & $0-1-2-3-4-5-6-7-8-9-10$ \\
\hline Tocar el balón durante el juego en ataque & $0-1-2-3-4-5-6-7-8-9-10$ \\
\hline
\end{tabular}


Señala lo que más te gusta hacer cuando JUEGAS UN PARTIDO (solo puedes marcar una)

LANZAR FINTAR OTROS PASAR DEFENDER
Señala lo que más te gusta hacer cuando ENTRENAS (solo puedes marcar una)

LANZAR FINTAR OTROS

PASAR DEFENDER

C1.-Señala en qué medida te gusta atacar y defender. Rodea con un CÍRCULO:

\section{ACCIÓN DE JUEGO}

EL ATAQUE

LA DEFENSA

\section{0 (NO ME GUSTA) 10 (ME GUSTA MUCHÍSIMO)}

$0-1-2-3-4-5-6-7-8-9-10$

$0-1-2-3-4-5-6-7-8-9-10$

¿Qué te gusta más atacar o defender? (Debes elegir solo una): DEFENDER ATACAR

Señala en qué medida te gusta jugar cada una de las siguientes situaciones. Rodea con un CÍRCULO:

\section{ACCIÓN DE JUEGO}

$1 \mathrm{x} 0$

$1 x$ Portero

$1 \times 1$

$2 \times 1$

$2 \times 2$

$3 \mathrm{X} 3$

Situación de juego con más jugadores
EN LOS ENTRENAMIENTOS 0 (NO ME GUSTA) 10 ( ME GUSTA MUCHÍSIMO)

$0-1-2-3-4-5-6-7-8-9-10$

$0-1-2-3-4-5-6-7-8-9-10$

$0-1-2-3-4-5-6-7-8-9-10$

$0-1-2-3-4-5-6-7-8-9-10$

$0-1-2-3-4-5-6-7-8-9-10$

$0-1-2-3-4-5-6-7-8-9-10$

$0-1-2-3-4-5-6-7-8-9-10$ 